\title{
Block Chain: Cryptographic Method in Fourth Industrial Revolution
}

\author{
Raihana Syahirah Abdullah ${ }^{\text {a* }}$, Faizal M.A. ${ }^{\text {b }}$ \\ ${ }^{\mathrm{ab}}$ Faculty of Information and Communication Technology, Universiti Teknikal Malaysia Melaka, Hang Tuah Jaya, \\ 76100 Durian Tunggal, Melaka, Malaysia \\ E-mail: raihana.syahirah@utem.edu.my
}

Received: 14 July 2018; Accepted: 07 October 2018; Published: 08 November 2018

\begin{abstract}
This paper has reviewed the blockchain domain that suits with the current Industrial Revolution (Industry 4.0) uses cryptographic method blockchain. The implementation of this new cryptographic method in Industry 4.0 is currently being used widely as it eases the process of financial transaction and other process that have been as issue related with cyber security. The collection of review papers shows that the blockchain technology has high potential to grow wider not just in financial technology services and manufacturing industry but also in public sector, health care and even media industry. The papers elaborate how the blockchain able to be applied in different fields of technology in order make a secure and more protected services without entrusted the third party to avoid vulnerability to be attacked and misused. Nevertheless, the applications of the blockchain in various field helps in making the industry revolution.
\end{abstract}

Index Terms-Blockchain, Industry 4.0, Machine to Machine, Cryptocurrency, Smart Contract, IoT mobile.

\section{INTRODUCTION}

The innovations through rapid development of technology from previous fragmented fields such as artificial intelligence (AI) in robotics and nanotechnology has leads us into Fourth Industrial Revolution, or also known as Industry 4.0. This revolution is breaking down the barriers between physical and virtual worlds to governance blockchain as the method of cryptographic method. The blockchain is a high-principled digital registry of private transactions with additional feature that not just record the financial transactions but also virtually everything of value [1].

Cryptocurrency like a Bitcoin, Ethereum and Monero has implement the blockchain technology. In the recent scenario, WannaCry had brought back the attention on cryptocurrency after using Bitcoin as a method of transaction to pay the ransom. Nonetheless, cryptocurrency not only had shown their potential in criminal scene, but the potential on recognized by few transaction power houses. The blockchain is a decentralized and immutable data storage without any interference from central authority as the network recheck for each other to provide the same data. A single change will not be validated unless all users in the network had also changed. Industry 4.0 also introduces the concept of machine-to-machine (M2M) communication, cyberphysical system (CPSs) and Internet of Things (IoT). $\mathrm{M} 2 \mathrm{M}$ is the ability of industrial components communicate with each other, CPSs is monitor physical processes and IoT is a system that connected computing devices, mechanical, objects, animals or humans that provided a unique identifier and able to transfer data over a network. For that purpose, the blockchain making its own platform for Industrial Internet of Things (IoT), where a model called Cloud-Based Manufacturing (CBM) is leveraging the IoT technologies. In a mean time, a decentralized peer-to-peer platform based on blockchain is proposed that known as BPIIoT. This platform enables the CBM to enhance its existing functionality on-demand access to manufacturing resources without the essential for a trusted intermediary [2].

Nowadays, the blockchain technology has been widely used in various application that involved with financial services as it offers advantages for the parties that records every daily transaction in a secure manner. One of the most famous topics among the researchers is applying the blockchain technology in the energy sector. Most of the financial transaction in the energy sector is centralized and still depends on the third party to process those transaction between the first and second party. This will cause disruption of the trading activities due to mistakes from the third party and this will affect the customer satisfaction.

However, few disruptive potentials had been known such as foreign exchange transfer and identity management, there are still an extent that can also improve the industries like travel management, intellectual property right in media and manufacturing. A decentralized energy trading system by using blockchain allows the prosumer and consumer to create a trading energy transaction via smart grid architectures [3]. Due to no third party involved during the transaction process in the decentralized system, the cost can be reduced and the quality of the service in the trading system will be in a whole new level. In this era, privacy has become the big concern for every user for their personal privacy that involved third party services. This paper addresses the 
users face as the privacy concerns especially on mobile platforms. The paper will review the protection against several common privacy issues as in data ownership, data transparency, auditability and fine-grained access control. These issues have reflected how the system follows the security principles [4].

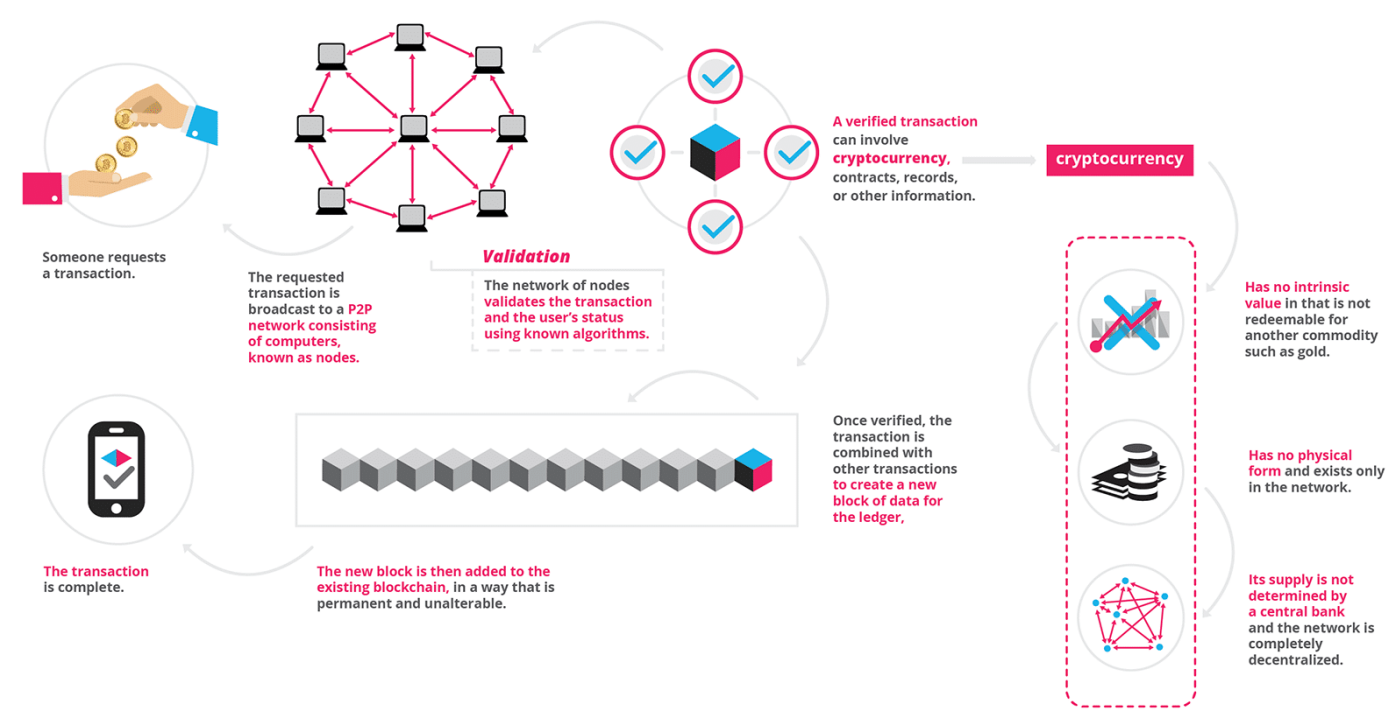

Fig.1. Simple Info graphic on Blockchain [1]

\section{RELATED WORK}

This industrial revolution rises along with the vulnerabilities within the industrial systems, which must be treated and managed for the extended the ecosystem to mitigate threats. This is where blockchain seems to shine brightly, to offer an innovation to Industry 4.0 cybersecurity risk management [5]. The Industry 4.0 is trying to achieve machines autonomy, where blockchain is seems to key between machines (M2M), as a medium of the records or registry. By implementing this technology, for example the cyber-physical system such as smart-factories, they can place an order autonomously and safely while optimizing the process and detecting malfunctions in the process. With the ability of allowing a frictionless and transparent financial transaction, blockchain make it looks necessary for the industrial revolution [14].

The paper by [6] is to explore application of blockchain technology related to the Industry 4.0 by presenting an example where blockchain is implemented to facilitate machine to machine in chemical industry. It eases the M2M commodity trading. Blockchain can hold any information and can set rules on how information is updated. It also known as block that append and link to the previous block using hash (content that running in question through a cryptographic hash function that can produce a hash for any input but difficult to derive the input). As a result, this paper was describing and discusses the research and application landscape of blockchain in detail. It also describes about technology that have been significant under-researched potential to support and enhance efficiency of the revolution.

In financial area, Bitcoin showed the possibility of decentralized network of peers accompanied by public ledger. As we already known, there is a rapid increment of data information in this era. We are now in this big data era where data is constantly being collected and analyzed that lead to the growth of innovation and economic. They even being collected to personalize services, optimize the corporate decision-making process and predict future trends. However, there has been many issues regarding the data privacy. So, we need to construct a privacy of personal data management platform by combining the blockchain and off-blockchain storage. The concept of decentralized energy trading through multi-signatures and the anonymous messaging stream has been proposed by [3]. This concept is applied in many fields. One of the possibilities in using blockchain technology is on identity management. Blockchain technology is proposed in giving a secure solution without any trusted, central authority. In managing individuals easier, blockchain is used to create an identity and enable them to control over their data confidentiality. The combining principle of decentralized blockchain with identity verification helps in creating a digital watermark that can be assigned to every online transaction [7].

Another paper in [4] discussed about the incidents of surveillance and security disclosure about user's privacy that involved third-parties' activities. It also demonstrated how Bitcoin has become an initiative in the financial space that shows a possibility of a trusted and auditable computing using a decentralized network of peers accompanied by a public ledger. Then, the implementation of a protocol which turns a blockchain into an automated access-control manager without involvement of third-party also has been describe. Private message and broadcast message are the two types of communication that been used in the system. If there is a disagreement or problem during the transactions that involved the parties, the Distribution System Operator 
(DSO) will be able to solve it. Next, the concept of green blockchain in handling the system was suggested by [8] that develop a green certificate in eco-area. Besides, a concept of distributed proportional-fairness control via blockchain smart contract has been shown by Danzi et. al. This system can control the output of energy by adjusting it based on requirements. Within the year of popularity, the newly found technology had few practical use cases and new application are continuously introduced. A survey was done in the World Economic Forum in 2015 that forecast $10 \%$ of global GDP will be stored in blockchain by 2027 .

Smart contracts or bundled of coded logic is a procedure what use the advantage of the ledger. If conditions of the contract are met, the agreed business operations will automatically proceed without any needs of human interaction. The benefits of these are reduction in transaction costs, improve transparency and efficiency in two key elements by removing a middle man. This application can ease intra-bank settlements by reducing the time and not yielding in quick assets transfer and reduce risks of counterparty not paying. Examples of the smart contract usage are Linq by [9] that allows actor on marketplace to set up confidential agreements.

In addition, blockchain technology has been proposed in a framework for access control in IoT which also enable users to own and control their data. The revolutionary evolution of IoT is focusing in security and privacy that will determined their success or failure subsequent. The proposed of a new framework named FairAccess is to give people that fairly belongs to them. The framework is bit different from financial bitcoin transaction which it introduces new types of transaction that are used for some activities grant, get, delegate and revoke access. Moreover, it is based on the main principles which a driven and transparency [10].

The blockchain technology was introduce with bitcoin. The bitcoin transaction was recorded in public ledger. There were divided in several areas that have been search. For explored area, it focuses on security and privacy. A comprehensive review by [11] found that most research publications are apply in this area. The issues are also addressed by [12] who describe two solutions to solve the problem of create a digital currency with the advantages of physical cash.

The blockchain in industrial platform (BPIIoT) proposed by [13] enables the development of different distributed apps (Dapps) for manufacturing. It has wider scope compared to existing solution called Slock. It can develop different decentralized and peer-to-peer manufacturing applications as shown in Fig. 2. Furthermore, BPIIoT platform consist a single-board computer including the cloud and blockchain bridges, sensors and actuators connectivity drivers, blockchain wallet, controller service, I/O and device management components and an interface board. The blockchain act as a distributed database that keep the records of transactions on a blockchain network. Blockchain also generally is maintained by a network of nodes and each node executes and records the same transactions. Moreover, the blockchain is duplicated among the nodes in the blockchain network. Apart from that, all the transactions can be read by any node in the network.

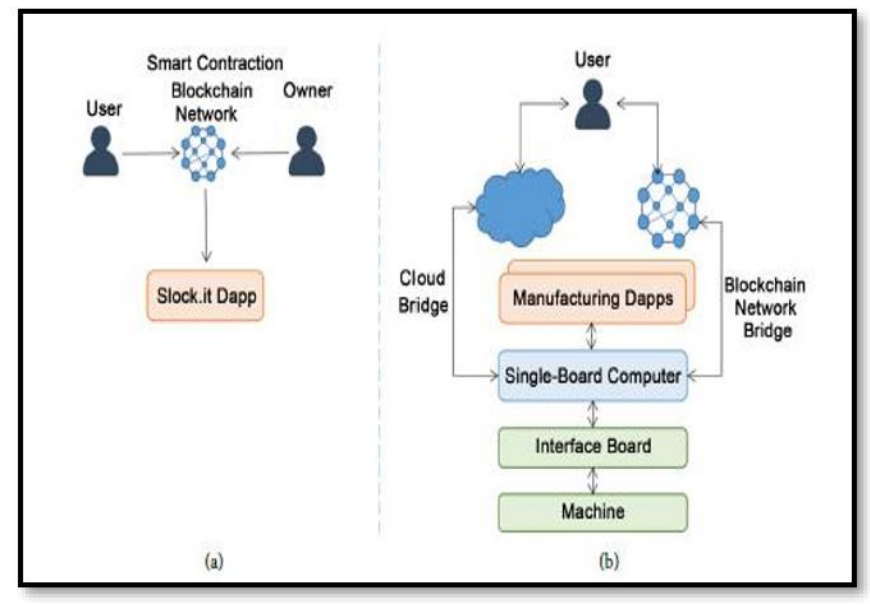

Fig.2. Slock.it vs BPIIoT [13]

In contrast to Industry 4.0, manufacturing industry will be a focus as a use case of blockchain. IBM and Maersk had implemented blockchain in logistic as a supply chain risk management and digital product memory. In PoC, blockchain is using to track containers during shipment as the goal is to reduce effort and paperwork that needed. In order improvise the traceability of certifications and other information's of product into the blockchain, the authenticity of a product by digital passport that can determine the origin. Provenance was using a blockchain to register every step of the production process in ensuring the transfer of ownership are explicitly authorized without having trust issues. Another company that trying to improvise trust issues is Everledger that extend blockchain to luxury goods. Everledger use the blockchain to register diamonds and secure the transaction history and ownership. Hence, the application of blockchain in IoT and Industry 4.0 is to solve the identification problem of IoT devices and minimize the vulnerability. Due to that, the company register the 
devices on a blockchain to create a device digital identity that cannot be manipulated. The advantage of this is information of the devices can be dynamically updated in comparison to traditional one. As the result, insurance if data not being manipulated afterwards and all standards can be achieved.

Blockchain technology also enables the creation of associated mechanism of smart contracts, with rules and regulations that both sides can specify, which is ideal for supply chain risk management. Smart contract is used to negotiate pricing for the design file to print by service provider. These steps are carried out without a middleman functionality. The knowledge of the transaction will increase the trust and enable large cost savings in terms of warranty, maintenance or recycling. Authentication of the part are enabled to helps technician ensure the counterfeit.

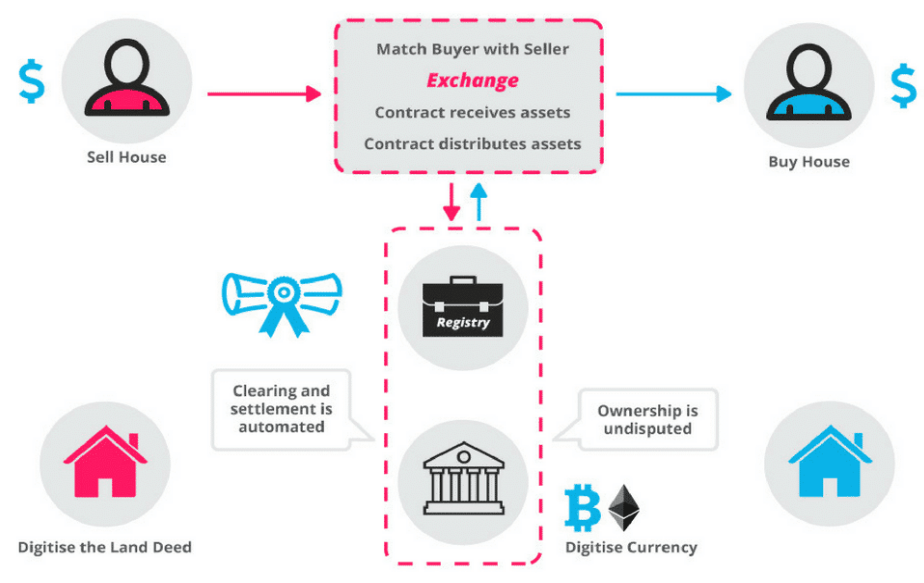

Fig.3. The Structure of Smart Contract Works

Throughout the paper proposed by [4] which focus on mobile platforms, the system made up of three entities which are mobile phone users, services and nodes which is trusted in maintaining the blockchain and its other elements for processing. These three entities set up a decentralizing system which shows how the system will figured out the privacy problem on mobile platforms.

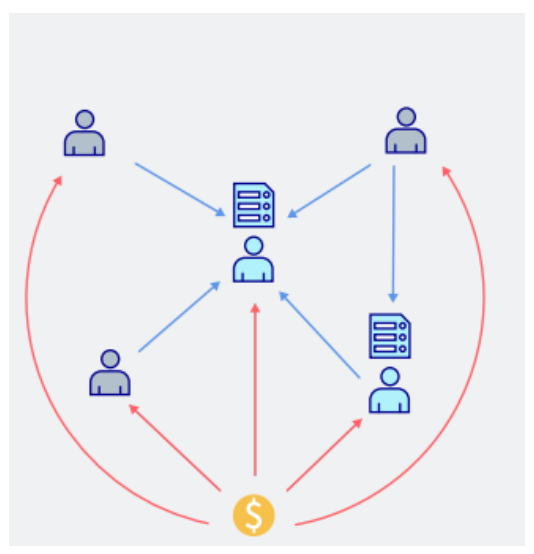

Fig.4. Overview of Decentralized Platform [4]

By applying the blockchain technology, it is possible to create a decentralized energy trading system between the neighbors via smart grid architecture. In this paper, we assume the smart grid has the ability to send and receive energy between prosumer and consumer [3]. The household that produce the renewable energy will transfer the energy to the neighbors in need. The decentralized energy trading system will ensure to supply the energy via smart grid whenever they receive a demand from the consumers. Meanwhile, the decentralized storage in the blockchain system will secure the information of the trading transaction between parties from attacker. The third-party involvement will no longer needed in the decentralized energy trading system in order to dealing with the traders during the energy trading activities. Instead the process will run automatically, and the energy will be sent after the miner solving the proof-of work and transmits to the network. The technology aims to become a local peer-to-peer retailer of renewable energy and become an energy exchange platform that can be used by the prosumer in order to sell their surplus energy without relies on the third party [3]. This system can act as a solution which combines security and transparency among the neighbors and able to measure the effectiveness of the blockchain technology applied in the system.

\section{ANALYSIS AND DISCUSSION}

Blockchain technology is basically some kind of new cryptographic method in dealing with process that associate with cryptocurrencies such as bitcoin, but also applicable to other fields and use cases including in securing Industry 4.0. The blockchain is also acting like a distributed database running on multiple servers, that store transactions record, where each new transaction is associated with the previous transaction, to build a chain. Each block of chain is checked for the security and integrity of each transaction or data entry by a few parties, making it immutable, verifiable and pseudonymized [5]. The power of the chain is the main structure of a blockchain. It provides smart industry a few applications such as to reduce cybersecurity risk. For example, supply 
chain risk management. This is because a high responsive supply chain is needed by those smart industry, but in cybersecurity, this extended supply chain is something to be concern of. According to Bomgar, when the thirdparty vendor use increase, the associated risks will also increase.

Blockchain technology has created a decentralized environment where the transactions and information didn't involve the third party. Model for decentralized energy trading among neighbors can be designed which uses a blockchain scheme. This process will allow the person who surplus energy to conduct activities with consumers and the transaction will be processed and confirmed by the miners. Thus, the renewable energy and blockchain are two technologies that are worth betting on and promising for the future [3]. In the future, electricity sharing would be available on the blockchain. Houses and premises would be able to save more electric energy and even share it among themselves. This is because different house or premises use different amount of energy. Therefore, those house or premises that produce excess energy could sell it to others, those who needs more energy than them. The transaction involve here is seems to be where blockchain can change decentralized power generation [13].

For example, in a community, let say that everyone is connected at local level. Every house in the community is given the same amount of energy, but excess production of energy could occur due to less usage in the house or maybe excess energy production from their own solar panel perhaps. They can sell the unused energy or excess energy to another neighbor. The grids are said to be connected to each other forming a virtual micro-grid, on the cloud that guarantees local production is consumed locally in a real-time energy exchange. Intelligent contracts (i-contracts) enable Blockchain to allow transaction between consumers. This method enable consumer to choose where to buy excess energy from and how much they want to buy, where the transactions are registered on the blockchain. Within the grid or blockchain network, the transactions are available to everyone.

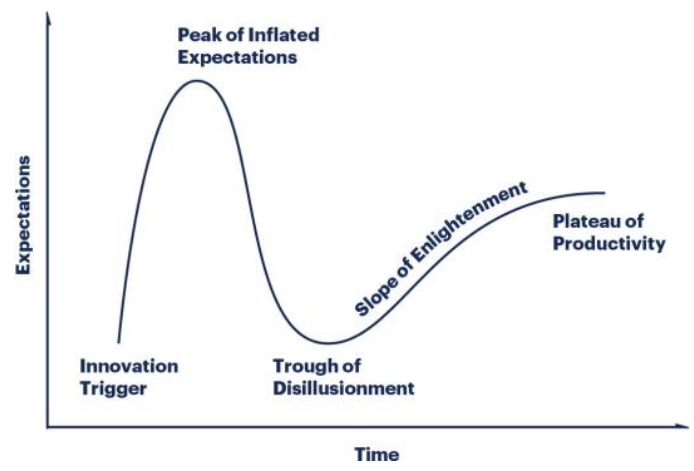

Fig.5. Gartner Hype Cycle

To prove the potentiality of blockchain application technology, the current phase of blockchain will be determined. The Gartner Hype Cycle is a tool that classify the maturity, adoption and social application of specific technologies will be use as illustration. The concept was developed by Gartner Inc, a IT firms to shows the expectations of technology over time. The cycle has 5 segments. After the technology triggered the enthusiasm increases dramatically with unrealistic expectations emerge. If the expectations cannot be achieved, the peak is passed, and expectations fall into trough of disillusionment. Increment number of people understands the technology will occur and the slope of enlightenment happens, and mainstream adoption takes place. In general, majority have mutual understanding that the hype will decrease in long-term but will not go down below half of the gradient. Once implemented, the likeliness of switching back to old technologies is far too less. While the hype is real, cautious steps should be taken.

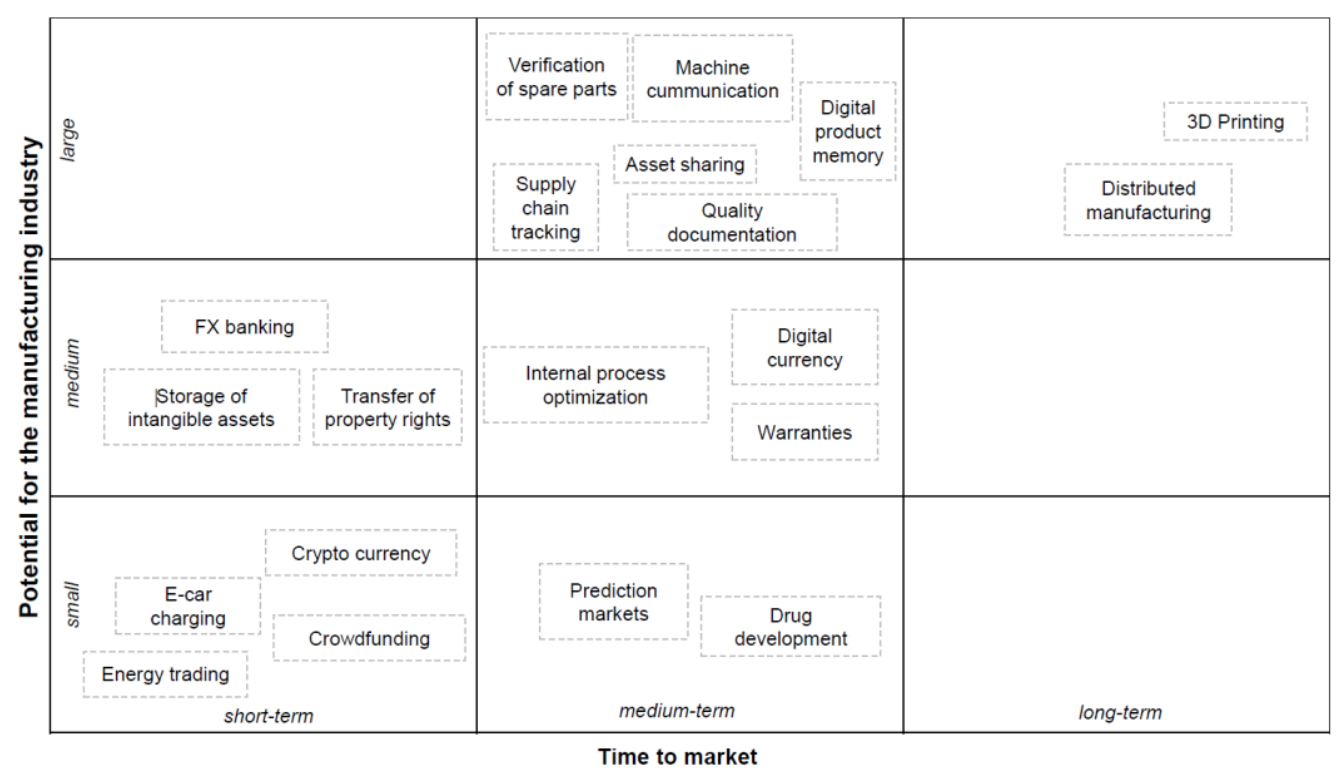

Fig.6. Blockchain Potential Application for Manufacturing 
Two dimensions were chosen to structure the application of blockchain that are "time to market" and "potential for the manufacturing industry". The reason of the first one is because most use cases are only proofs of concept and at the beginner stage before entering the market entry as use cases are more driven by hype without chances of implementation. The second is because not many applications and these have different potentials for a significant impact. Nonetheless, taking count the manufacturing industry is widen cases, some use cases might be transferable from other industries (cross-potential). Asset sharing are useful in most industries and companies in manufacturing industry have higher chances because large energy consumption as a global company that need cost-efficient treasury system.

Leading researchers and industry experts predict that first stage of the use cases will be implemented and announced to public within the next three to five years from now. The time taken to marketize the application differs remarkably among the key areas, due to different technical requirement for the specific solution. However, in the process of implementation by financial sector, few industries are also trying to seize their development rapidly. Nonetheless, technical barriers had greatly affected the time for the blockchain to be use commercially [14]. Majority of the experts states the challenges as solvable in the future. This clearly seen in limited scalability problem faced by IoT and the manufacturing industry, yet a breakthrough by evolving concept IOTA shows positive sign for the solution. Additional challenge problems that need to be solve are from energy sector that need regulation on the establishment of a blockchain based energy platform and legal issues of liability in the 3D printing. Surprisingly, all the experts agreed independently that the main problem is the readiness on accepting this innovative technology.

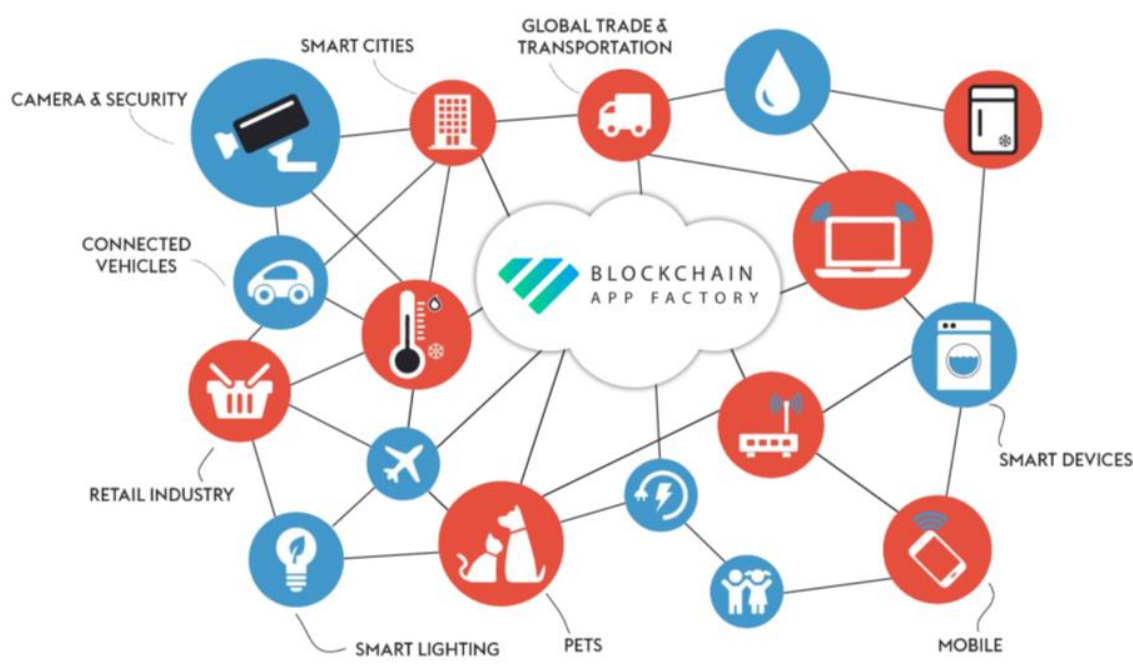

Fig.7. Blockchain Implementation of IoT Device

A demonstration on a smart contract for machine service and part replacements is set up as to make an agreement between the machine and the service to place a part replacement order or schedule a machine service request. The implementation is done using the Python which runs on the IoT device (Beaglebone Black singleboard computer). Based on the Fig. 7, it showed how the controller service continually monitors the temperature and vibration levels of different parts of machine. The placement of the smart contract on Ethereum blockchain network allows the assignation of the address to the contract.

The mobile platform decentralized system is analyzed based on its protocols [15]. Protocol 1 symbolize the identities, thus shows the implementation for a single owner (user) and single guest (service). The identity is consisting of signing key-pairs for the owner and guest and also a symmetric key that is used to encrypt and decrypt the data as to make it secure from other players in the system. Protocol 2 illustrate the auxiliary functions which verifies the authorized access given to the originator. While protocol 3 and protocol 4 is the core protocols executed on the blockchain. Protocol 3 is executed when a Taccess transaction is received and Protocol 4 will be executed for Tdata transactions. Fig. 9 shows the architecture of trading renewable energy system in the peer-to-peer network. There are four prosumers involved that have the surplus energy and they willing to sell their energy to the neighbors in need. When the prosumer wants to sell the energy, he/she will announce to the auction board which contains information about the amount of energy they want to sell and the price. There is a party in the network named Distributed System Operator (DSO) that will solve any disagreement occur between the prosumer and consumer during the trading activities.

In order to get the data communication results, some component will be added into the network and for every updated data to the new block, the miner should solve the proof-of-work and then propagated that data to the entire network. There are five components in the network which are router, local computer, laptop, switch and wireless 
router. In this system, the router will transmit the data of transaction from source to another computer in the peerto-peer as shown in figure 8 .

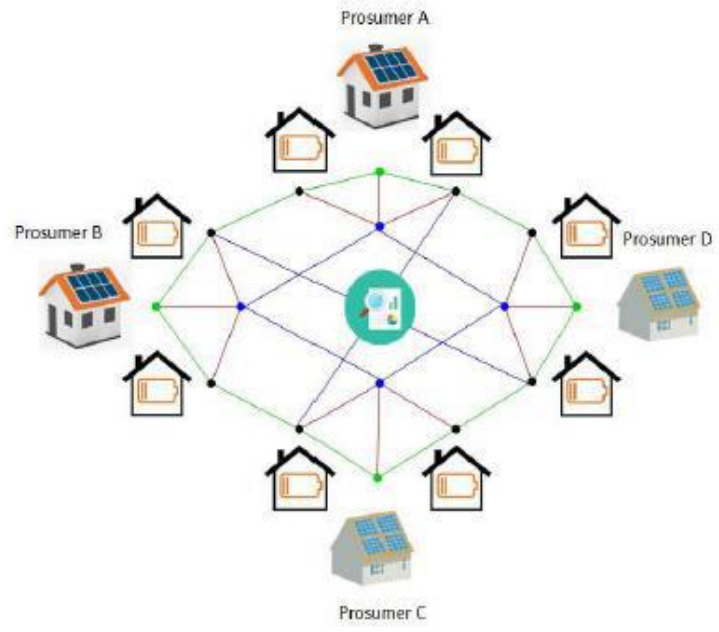

Fig.8. Decentralized Energy Trading System

Based on the experts and market survey, the use cases of blockchain technology had been identified. These use cases were analyzed by using cluster analysis and evaluated by criteria of beneficial application of blockchain. The result assessed the potential use cases and time until market entry. Furthermore, the cluster was used to identify cross-potentials that shows the relevant ness in few industries. First market-ready solutions will start to show in the next three to five years and it is suggested that the application most likely from the financial industry. Referring to the Gartner Hype Cycle, the hype is currently at the peak or short before. Thus, a slope of excitement for the next couple of years should be predicted. A major reason for this is the technical and cultural challenges that need a significant amount of breakthrough, thus it is believed to be solvable in the future and the technology will become a key to transform many industries.

The BPIIoT platform implementation for the IoT devices seem can be improved in future by focusing on implementation and demonstration to give more realistic solutions such as on demand manufacturing and device self-service. The future extensions in the mobile platform decentralization could be applied in this system as to shape mature distributed trusted computing platforms. A secure Multi-Party Computation (MPC) could be used in future to evaluate any function securely. Combining the MPC to work with blockchains in this framework, able to make the data for the final result on the public ledger, but safe against tampering.

For the example, a mobile application is developed to allow a secret voting for an election in a city which is augmented with the MPS capabilities. This implementation has leads to the result where no one can learn for the individual votes however the can only see the results of the election. This happens when the city submits the back-end code after the online election at random and an interpreter transform the code into a secure MPC protocol. That's how a better approach for the decentralized blockchain in this platform is planning to be achieved.

As to improve the trust and decision-making in blockchain, a new measure can be defined based on node behavior, where we could set the trust of each node as the expected value of it behaving well. This new dynamic measure is using similar reasoning as before which is known as Proof-of-Work reasoning, where it causes the nodes to pour important sources into the system are less likely to be deceiving. The components in the network architecture plays a main role in supporting the continuity of a system and it has the input and output gateway of data blocks. The components will require knowledge of data blocks location and also the ability to fetch data blocks in appropriate node that contains requested data in order to be in charge of distributing the record of data transaction in the network. In order to manage and control the input and output in the network, assume there is a node manager component which follow the rules of the chord protocol [3]. When a node wants to be inserted in the network, the component will generate a new identifier based on the protocol and will notify the other node to make it more accessible. Once the transaction of data has been successfully inserted into the block, the record of data become protected.

Table 1. Benefits of Applying Blockchain Technology for Certain Application in Manufacturing

\begin{tabular}{|l|c|c|c|c|}
\hline & $\begin{array}{c}\text { Multiple } \\
\text { parties share } \\
\text { data }\end{array}$ & $\begin{array}{c}\text { Multiple } \\
\text { parties } \\
\text { update data }\end{array}$ & $\begin{array}{c}\text { Require-ment of } \\
\text { verification }\end{array}$ & $\begin{array}{c}\text { Intermedi-aries } \\
\text { add cost and } \\
\text { complexity }\end{array}$ \\
\hline 3D printing & $\checkmark$ & & $\checkmark$ & $\checkmark$ \\
\hline Asset sharing & $\checkmark$ & $\checkmark$ & $\checkmark$ & \\
\hline Digital product memory & $\checkmark$ & $\checkmark$ & $\checkmark$ & $\checkmark$ \\
\hline Distributed manufacturing & $\checkmark$ & $\checkmark$ & $\checkmark$ & $\checkmark$ \\
\hline Machine communication & $\checkmark$ & $\checkmark$ & & $\checkmark$ \\
\hline Quality documentation & $\checkmark$ & $\checkmark$ & $\checkmark$ & $\checkmark$ \\
\hline Supply chain tracking & $\checkmark$ & $\checkmark$ & $\checkmark$ & $\checkmark$ \\
\hline Verification of spare parts & $\checkmark$ & $\checkmark$ & $\checkmark$ & \\
\hline
\end{tabular}


The paper published by past researchers demonstrates that it is possible to success implement the blockchain technology. Blockchain technology has the potential to revolutionize the engineer industry by facilitate the transition and function of industry 4.0. For the further innovation, remains many unexplored research area and application areas. Decentralized system for mobile platform has enable user not to entrust third party in any of their transaction. Besides, the solution of blockchain as an access-control moderator with off-blockchain storage able to provide users to own and control their data without affecting the security and ability of companies while providing personalized services.

Generally, decentralized system in either mobile platform or IoT platform, showed that the blockchain network is maintained by a network of nodes. Then, every nodes are able to records the same transaction occurred. Therefore, every transaction are verified and validated by an agreement among the peers without trusting each other. Furthermore, there are many advantages of using this decentralized platform. When it comes to collecting, storing and sharing sensitive data, the laws and regulation can be simpler as it can be programmed into the blockchain itself, thus they are applied automatically. Another advantage could be the capability of being a tamper-proof data. As in other situation, the ledger can be treated as legal proof when accessing data.

Apart from that, the advantages of blockchain in decentralized system is the blockchain is autonomous, specifically when dealing with IoT devices [16]. Blockchain allows the devices to communicate to make transaction autonomously since each of the device has its own blockchain account, again without entrusting the third-party. Besides, the strong cryptography that featured in the blockchain gives advantage for it to be secure and auditable for everyone on the network where they are able to know all about the transactions. This is because, blockchain network has maintained the transparent nature of the public ledger and all the transactions are not able to be disputed.

\section{CONCLUSION}

The blockchain technology that have been applied in the system allows the parties to manage the trading activities between the neighbors. Hence, the decentralized energy trading system will create a better future in order to provide a clean energy for the neighbors in need. However, the blockchain structure to spread the data by using the network is still exposed to numerous attacks. Therefore, in the future, more strategies needed in order to prevent the system from attacks. In a nutshell, all the applications of the blockchain in various field helps in making the industry revolution. The vulnerabilities of the data collected is safer when no more third-party is being entrusted while having the blockchain network. Nevertheless, the lack of awareness regarding this technology has affect its widespread adoption.

\section{ACKNOWLEDGEMENTS}

The authors would like to express the appreciation to Inforsnet Group Research of Universiti Teknikal Malaysia Melaka (UTeM) in encouraging the authors to publish this paper.

\section{REFERENCES}

[1] Don \& Tapscott Alex. (2016). What is Blockchain Technology? A Step-by-Step Guide for Beginners. Retrieved April 3, 2018, from https://blockgeeks.com/guides/what-isblockchaintechnology/

[2] Bahga, A., \& Madisetti, V. K. (2016). Blockchain platform for industrial Internet of Things. Journal of Software Engineering and Applications, 9(10), 533.

[3] Rahmadika, Sandi. (2018). The Noble Approach of Blockchain Technology for Energy Trading System and Security Issues.

[4] Zyskind, G., \& Nathan, O. (2015, May). Decentralizing privacy: Using blockchain to protect personal data. In Security and Privacy Workshops (SPW), 2015 IEEE (pp. 180-184). IEEE.

[5] Gozzi Alexandre. (2018). How Industry 4.0 uses Blockchain Technology - Blog Sopra Steria. Retrieved April 3, 2018, from https://blog.soprasteria.com/industryblockchaintechnology/

[6] H. Watanabe, S. Fujimura, A. Nakadaira, Y. Miyazaki, A. Akutsu, and J. J. Kishigami. Blockchain contract: A complete consensus using blockchain. In 2015 IEEE 4th Global Conference on Consumer Electronics (GCCE), pages 577-578, Oct 2015. doi:10.1109/GCCE.2015.7398721.

[7] M. English, S. Auer, and J. Domingue. Block Chain Technologies \& the Semantic Web: A Framework for Symbiotic Development. Technical report, University of Bonn, Germany, May 2016.

[8] F. Imbault, M. Swiatek, R.de Beaufort and R. Plana, "The Green Blockchain Managing Decentralized Energy Production and Consumption", IEEE International Conference on Environment and Electrical Engineering (EEEIC/ I\&CPS Europe), 2017.

[9] P. Danzi, A. Marko, C. Stefanovic, and P. Popovski, "Distributed ProportionalFairness Control in Microgrids via Blockchain Smart Contracts", arXiv: 1705.01453v2 [cs.MA], 2017

[10] Ouaddah, A., Abou Elkalam, A., \& Ait Ouahman, A. (2016). FairAccess: a new Blockchain - based access control framework for the Internet of Things. Security and Communication Networks, 9(18), 5943-5964.

[11] J. Yli-Huumo, D. Ko, S. Choi, S. Park, and K. Smolander. Where Is Current Research on Blockchain Technology? - A Systematic Review. PLoS ONE, 11(10): $1-27, \quad 10 \quad 2016$. doi:10.1371/journal.pone.0163477

[12] N. Koblitz and A. J. Menezes. Cryptocash, Cryptocurrencies, and Cryptocontracts. Des. Codes Cryptography, 78(1):87-102, Jan. 2016. ISSN 09251022. doi:10.1007/s10623-015-0148-5.

[13] Bansal Jaideep. (2017). some villages are embracing the Fourth Industrial Revolution faster than cities. This is how | World Economic Forum. Retrieved April 3, 2018, from https://www.weforum.org/agenda/2017/11/t heindian-villages-embracing-the-fourthindustrialrevolution 
[14] Mezni Mehdi. (2018). Blockchain, and Industry 4.0: a closer look. Retrieved April 23, 2018, from https://en.decentral.news/2018/01/26/blockc hainindustry-4-0

[15] N.Z. Aitzhan and D. Svetinovic, "Security and Privacy in Decentralized Energy Trading through MultiSignature, Blockchain and Anonymous Messaging Streams", IEEE Transactions on Dependable and Secure Computing, 2016

[16] M. O'Dair, Z. Beaven, D. Neilson, R. Osborne, and P. Pacifico. Music on the Blockchain. Technical Report 1, Blockchain for Creative Industries Research Cluster, Middlesex University, UK, July 2016.

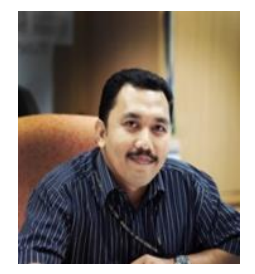

Mohd Faizal Abdollah are currently a senior lecturer in Universiti Teknikal Malaysia Melaka. Their research area are IDS, Malware, Forensic and Network Security.

\section{Authors' Profiles}

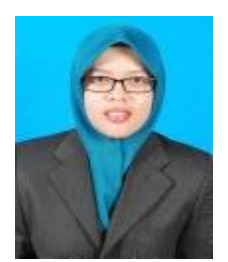

Raihana Syahirah Abdullah are currently a senior lecturer in Universiti Teknikal Malaysia Melaka. Their research area are IDS, Malware, Forensic and Network Security.

How to cite this paper: Raihana Syahirah Abdullah, Faizal M.A.,"Block Chain: Cryptographic Method in Fourth Industrial Revolution", International Journal of Computer Network and Information Security(IJCNIS), Vol.10, No.11, pp.9-17, 2018.DOI: 10.5815/ijcnis.2018.11.02 